\title{
A Unified Approach to Orthogonal Digital Filters and Wave Digital Filters, Based on LBR Two-Pair Extraction
}

\author{
P. P. VAIDYANATHAN, MEMBER, IEEE
}

\begin{abstract}
The LBR-extraction approach is extended in order to derive wave digital filters and several orthogonal digital filters in a unified manner. The derivation clearly places in evidence the underlying orthogonality property of all these structures, which can therefore be implemented based on a simple building block, namely, the "planar rotation" operator. The derivation directly emphasizes the concept of "structural boundedness" as a requirement for low sensitivity. In addition to wave and orthogonal filters, a number of other methods for forcing structural boundedness are indicated.
\end{abstract}

\section{INTRODUCTION}

$\mathrm{W}$ AVE digital filters [1]-[4] and orthogonal digital filters [5] are two well-established families of digital filter structures, and are known for several excellent properties, under finite-precision constraints. Among these are low passband-sensitivity, stability in spite of parameter quantization, and possibility of achieving freedom from limit cycles that normally arise due to signal quantization. Wave digital filters are the result of a pioneering development in 1971 by Fettweis [1], who showed that, by systematically transforming a doubly terminated $L C$ network into the discrete-time domain, low-sensitivity digital filters can be designed. Since then, there has been considerable activity in the wave filter area, leading to a complete understanding of this successful class of filters.

The Gray-Markel normalized digital filter structures [6] are based on a tapped cascade of "orthogonal building" blocks. The recursive part of a transfer function is, therefore, realized by means of an orthogonal filter. A wide family of truly orthogonal digital filters has been developed in the classic work by Dewilde et al. [5], based entirely on $z$-domain concepts. This remarkable family shares a number of important properties with the wave digital filters. In addition, the normalized lattice structures [6] are known to be a form of wave digital filters [28].

Henrot and Mullis [7] have shown that a bichannel version of the Levinson's recursion algorithm can be used to derive a new class of orthogonal digital filters. The building blocks that result in their synthesis are extensions of the normalized lattice [6]. These filters possess many of the excellent properties that conventional orthogonal filters

Manuscript received September 4, 1984; revised November 22, 1984. This work was supported in part by the National Science Foundation under Grant ECS 84-04245.

The author is with the Department of Electrical Engineering, California Institute of Technology, Pasadena, CA 91125. have, and are in addition, simultaneously cannonic and "pipelineable." Related structures and new ones are proposed in [8] in an independent manner, and new results are obtained relating to the actual implementation of these structures in VLSI.

In a recent contribution [9], it was shown that wave digital filters can be synthesized independently in the $z$ domain without starting from an $L C$ prototype network. The purpose of this paper is to extend these ideas and obtain a unified derivation of wave filters and the various types of orthogonal filters outlined above. The "cordic processor" which implements a planar-rotation is found to be basic to both of these families, and these and related conclusions are obtained here based on the LBR-extraction approach [9]. The concept of "structural boundedness," which is key to the low-sensitivity property, is emphasized in this paper. All the structures presented here essentially indicate various ways of forcing this "boundedness."

The lossless bounded real (LBR) property, which is key to all our developments, is the $z$-domain version of a basic property satisfied by scattering matrices of lossless twoports in the continuous-time domain [10]-[12]. In the continuous-time domain, the scattering matrix is derived from an impedance matrix (satisfying the lossless positive real (LPR)) property, and is, therefore, a square matrix. However, an independent definition of the LBR property can be used even for rectangular matrices and, in particular, column vectors, which helps us to generalize the LBR approach for single-input multi-output situations.

In Section II we review certain preliminaries that will be required in later developments. Sections III and IV deal with the link between the LBR-approach, wave digital filters, and the orthogonal filters. The concept of "orthogonality" is shown to be generic to all these filters, enabling us to implement them in terms of planar-rotations. In Section V, the LBR-approach is used for an independent derivation of the pipelineable orthogonal filters introduced in [7] and [8]. Finally, in Section VI we indicate a number of other methods for incorporating structural boundedness in an implementation.

An interesting family of structures advanced by Vaughan-Pope and Bruton [27] should also be mentioned in the context of this paper; this class of structures is also based on ladder topology and has excellent sensitivity 


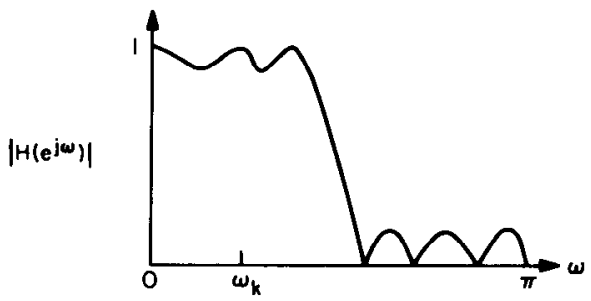

Fig. 1. A typical magnitude-response.

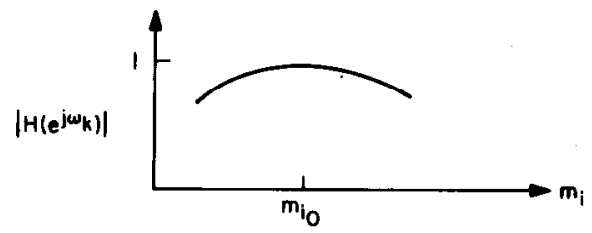

Fig. 2. Pertaining to (2).

properties. However, we will not have occasion to discuss this family in this paper.

\section{Review of Preliminaries}

The main purpose of this section is to review a number of concepts and definitions that will be used in the rest of the paper.

\section{Review of Structural Boundedness and Low Sensitivity}

Consider a rational scalar transfer function with real coefficients:

$$
H(z)=\frac{a_{0}+a_{1} z^{-1}+\cdots+a_{N} z^{-N}}{1+b_{1} z^{-1}+\cdots+b_{N} z^{-N}} .
$$

Let us assume that the structure implementing $H(z)$ has the following property: As long as the values of the multipliers are within a certain permissible range, the magnitude $\left|H\left(e^{j \omega}\right)\right|$ is bounded above by a scalar constant, say unity. (The precise nature of this "permissible range" depends upon the actual structure. In some of our examples, this range is $(0,1)$, while in others, this range is $(-1,1)$.) Thus the structure imposes a kind of "boundedness" or "passivity" on the transfer function. Let us consider a typical lowpass response, as shown in Fig. 1. At a frequency $\omega_{k}$ in the passband, the transfer function magnitude is precisely unity, for an infinite-precision implementation. If now an internal multiplier is quantized, then the magnitude $\left|H\left(e^{j \omega_{k}}\right)\right|$ cannot increase, because of the "structural boundedness." As a result, $\left|H\left(e^{j \omega_{k}}\right)\right|$ plotted against an internal multiplier $m_{i}$ has the form shown in Fig. 2. It is, therefore, clear that

$$
\left.\frac{\partial\left|H\left(e^{j \omega_{k}}\right)\right|}{\partial m_{i}}\right|_{m_{i}=m_{i_{0}}}=0 .
$$

In other words, the structural boundedness forces zerosensitivity property with respect to each internal multiplier at certain frequencies $\omega_{k}$ in the passband. Recall that structural boundedness, which implies

$$
\left|H\left(e^{j \omega}\right)\right| \leqslant 1, \quad \forall \omega
$$

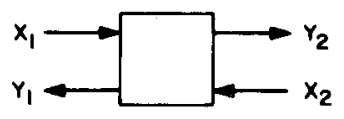

Fig. 3. A digital two-pair.

is equivalent to the property

$$
\sum_{n=-\infty}^{\infty}|y(n)|^{2} \leqslant \sum_{n=-\infty}^{\infty}|x(n)|^{2}
$$

for every square-summable (i.e., finite energy) input sequence $x(n)$. (This follows by invoking Parseval's relation. See [9].) Thus for a "structurally bounded" or "passive" implementation, the output energy is at most equal to the input energy. This kind of a boundedness property holds in a number of well-known structures, for example, the wave filters, the orthogonal digital filters, and the digital lattice filters. Apart from low sensitivity, there are several other consequences of this boundedness, as detailed in [9],[13]. This kind of structural boundedness is a general concept and can even be used for the design of low-sensitivity FIR filters [14]. Note that the bound of unity in (3) is only a convention. An arbitrary fixed constant, independent of the multiplier values, is in fact a sufficient bound.

With this motivation for boundedness, one can define a bounded-real function (BR) $H(z)$ to be any stable ${ }^{1}$ transfer function that is real valued for real $z$, and such that the condition of (3) is satisfied. A lossless bounded real (LBR) function is a BR function with strict equality in (3) for all $\omega$. Such a function is nothing but a stable all-pass function.

\section{Digital Two-Pairs}

In order to proceed further with our discussion on low-sensitivity filters, let us extend the above boundedness concepts to a "digital two-pair." A digital two-pair [15], shown in Fig. 3, is a two-input two-output system, described either by the chain parameters:

$$
\left[\begin{array}{l}
X_{1}(z) \\
Y_{1}(z)
\end{array}\right]=\left[\begin{array}{ll}
A(z) & B(z) \\
C(z) & D(z)
\end{array}\right]\left[\begin{array}{l}
Y_{2}(z) \\
X_{2}(z)
\end{array}\right]
$$

or the transfer parameters:

$$
\left[\begin{array}{l}
Y_{1}(z) \\
Y_{2}(z)
\end{array}\right]=\left[\begin{array}{ll}
T_{11}(z) & T_{12}(z) \\
T_{21}(z) & T_{22}(z)
\end{array}\right]\left[\begin{array}{l}
X_{1}(z) \\
X_{2}(z)
\end{array}\right] .
$$

A "reciprocal" two-pair satisfies the condition $T_{12}(z)=$ $T_{21}(z)$ or equivalently, $A D-B C=1$. The descriptions of (5) and (6) are related as indicated in [15]. The chain matrix of (5) and the transfer matrix of (6) are denoted by $\Pi(z)$ and $\tau(z)$, respectively.

A digital two-pair is said to be LBR if (a) it is stable, (b) $\tau(z)$ is real-valued for real $z$, and (c) $\tau^{t}\left(z^{-1}\right) \tau(z)=\boldsymbol{I}$. Condition (c), which is called paraunitariness, essentially

\footnotetext{
${ }^{1}$ In this paper, "stable" refers to bounded-input bounded-output stability, i.e., all poles are strictly inside the unit circle.
} 


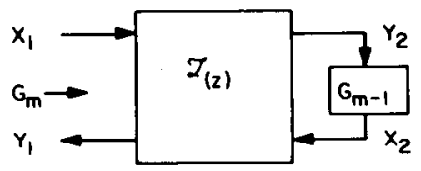

Fig. 4. Two-pair extraction.

implies

$$
\begin{aligned}
\sum_{n=-\infty}^{\infty}\left[\left|x_{1}(n)\right|^{2}+\left|x_{2}(n)\right|^{2}\right] & \\
& =\sum_{n=-\infty}^{\infty}\left[\left|y_{1}(n)\right|^{2}+\left|y_{2}(n)\right|^{2}\right]
\end{aligned}
$$

i.e., the energy of the output (vector) sequence is equal to that of the input (vector) sequence for every finite-energy input. In terms of the chain parameters, paraunitariness is equivalent to: ${ }^{2}$

$$
\begin{aligned}
1+\tilde{C} C & =\tilde{A} A \\
1+\tilde{B} B & =\tilde{D} D \\
\tilde{C} D & =\tilde{A} B .
\end{aligned}
$$

A stable reciprocal two-pair is LBR if and only if

$$
\begin{aligned}
A & =\tilde{D} \\
B & =\tilde{C} \\
A D-B C & =1 .
\end{aligned}
$$

Given a transfer function $G_{m}(z)$, the "extraction" of a digital two-pair leads to a remainder $G_{m-1}(z)$ (see Fig. 4) where $G_{m}(z)$ and $G_{m-1}(z)$ are related by the extraction formula:

$$
\begin{aligned}
G_{m-1} & =\left(C-A G_{m}\right) /\left(B G_{m}-D\right) \\
G_{m} & =\left(C+D G_{m-1}\right) /\left(A+B G_{m-1}\right) .
\end{aligned}
$$

Here the parameters $A(z), B(z), C(z), D(z)$ are the chain parameters as in (5). The subscripts $m$ and $m-1$ do not necessarily stand for order. Thus unless the two-pair is properly chosen, the order of $G_{m-1}$ is not less than that of $G_{m}$.

A digital filter transfer function can be synthesized [9] in a structurally bounded manner by realizing it as a cascade of LBR two-pairs, terminated in a BR multiplier $m_{0}$ (i.e., $\left.\left|m_{0}\right| \leqslant 1\right)$. Indeed, this also leads to an alternative approach to the synthesis of wave digital filters among others, without starting from an $L C$-prototype network. It has been shown by Fettweis [29] that the actual transfer functions of interest in a wave digital filter do not have zero sensitivity at the points of maximum power transfer in the passband. (The author wishes to thank a reviewer for bringing [29] to his attention.) However, Fettweis also points out in [29] that the nonzero component of this sensitivity is a constant independent of frequency, and, therefore, does not cause any concern at all in an actual implementation. To be more specific, the actual transfer function of interest $H(z)$ is in the form $H(z)=\alpha H_{1}(z)$ where $H_{1}(z)$ is such that $\left|H_{1}\left(e^{j \omega}\right)\right|$

\footnotetext{
${ }^{2}$ Superseript tilde indicates transposition followed by, replacement of $z$ with $z^{-1}$.
}

has zero sensitivity at points of maximum power transfer. The quantity $\alpha$ is a constant depending upon internal multipliers $m_{i}$, but as $\partial \alpha / \partial m_{i}$ is independent of frequency, the "sensitivity properties" of $H(z)$ and $H_{1}(z)$ are essentially identical.

We now extend the concept of two-pairs to the case where the scalar signals $X_{1}, Y_{1}, Y_{2}, X_{2}$ in Fig. 3 are replaced with vector signals $X_{1}, Y_{1}, Y_{2}, X_{2}$. Thus

$$
\left[\begin{array}{l}
X_{1}(z) \\
Y_{1}(z)
\end{array}\right]=\left[\begin{array}{ll}
A(z) & B(z) \\
C(z) & D(z)
\end{array}\right]\left[\begin{array}{l}
Y_{2}(z) \\
X_{2}(z)
\end{array}\right]
$$

and the transfer parameters $\boldsymbol{T}_{i j}$ are defined accordingly:

$$
\left[\begin{array}{l}
Y_{1}(z) \\
Y_{2}(z)
\end{array}\right]=\left[\begin{array}{ll}
T_{11}(z) & T_{12}(z) \\
T_{21}(z) & T_{22}(z)
\end{array}\right]\left[\begin{array}{l}
X_{1}(z) \\
X_{2}(z)
\end{array}\right]
$$

The parameters are related as

$$
\begin{aligned}
& T_{11}=C A^{-1} \\
& T_{12}=D-C A^{-1} B \\
& T_{21}=A^{-1} \\
& T_{22}=-A^{-1} B
\end{aligned}
$$

and

$$
\begin{aligned}
& A=T_{21}^{-1} \\
& B=-T_{21}^{-1} T_{22} \\
& C=T_{11} T_{21}^{-1} \\
& D=T_{12}-T_{11} T_{21}^{-1} T_{22} .
\end{aligned}
$$

Such two-pairs for which some or all of the chain parameters are matrices or vectors will be called matrix two-pairs. Clearly, the above description is meaningful only if $\boldsymbol{A}$ and $T_{21}$ are square, i.e., only if the vectors $X_{1}$ and $Y_{2}$ have the same number of components. For such cases, the "extraction formula" now becomes

$$
\begin{aligned}
\boldsymbol{G}_{m-1} & =\left(\boldsymbol{D}-\boldsymbol{G}_{m} \boldsymbol{B}\right)^{-1}\left(\boldsymbol{G}_{m} \boldsymbol{A}-\boldsymbol{C}\right) \\
\boldsymbol{G}_{m} & =\left(\boldsymbol{C}+\boldsymbol{D} \boldsymbol{G}_{m-1}\right)\left(\boldsymbol{A}+\boldsymbol{B} \boldsymbol{G}_{m-1}\right)^{-1} .
\end{aligned}
$$

A matrix LBR two-pair is defined in exactly the same manner as an LBR two-pair. In particular, the paraunitary property is equivalent to

$$
\begin{aligned}
\tilde{C} C+I & =\tilde{A A} \\
\tilde{B} B+I & =\tilde{\boldsymbol{D} D} \\
\tilde{C} \boldsymbol{D} & =\tilde{\boldsymbol{A} B} .
\end{aligned}
$$

A proof of (11)-(15) is included in Appendix A.6.

In this paper, in addition to the conventional two-pair, we are also interested in those matrix two-pairs with $X_{1}$ and $Y_{2}$ scalar, and with $Y_{1}$ and $X_{2}$ vectors of 2 components each. The two-pair extraction scheme for such twopairs is shown in Fig. 5, where $\boldsymbol{G}_{m}(z)$ and $\boldsymbol{G}_{m-1}(z)$ are clearly 2-component vectors. The function $G_{m}(z)$ is said to be BR if (a) each entry is stable, (b) $\boldsymbol{G}_{m}(z)$ is real for real $z$, and (c) $\boldsymbol{G}_{m}^{t}\left(e^{-j \omega}\right) \boldsymbol{G}_{m}\left(e^{j \omega}\right) \leqslant 1$ for all $\omega$. Such a function is LBR or allpass if the inequality in (c) holds with equality 


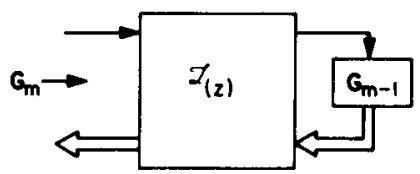

Fig. 5. Matrix two-pair extraction.

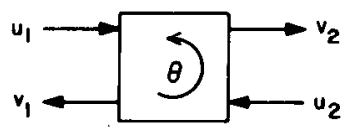

Fig. 6. The planar-rotation building block.

for all $\omega$. For a BR $G_{m}(z)$, it can be shown that: ${ }^{3}$

$$
\boldsymbol{G}_{m}^{\dagger}(z) \boldsymbol{G}_{m}(z) \leqslant 1, \quad \text { for all } z \text { with }|z|>1 \text {. }
$$

When $G_{m}(z)$ is a scalar, this follows from the maximum modulus principle. For vector $\boldsymbol{G}_{m}(z)$, a proof is given in Appendix A.1.

Next, referring to Fig. 4, if $G_{m-1}$ is (lossless) BR, then so is $G_{m}$ provided the extracted two-pair is LBR. Same is true with Fig. 5. The converse, however, is not necessarily true. Thus if $G_{m}$ is LBR and an "inappropriate" LBR two-pair is extracted, then $G_{m-1}$ might turn out to be non-BR (or even unstable). However, if $\boldsymbol{G}_{m}$ is LBR and an LBR two-pair is extracted, $G_{m-1}$ is always paraunitary, i.e., $G_{m-1}^{t}\left(z^{-1}\right) G_{m-1}(z)=1$ for all $z$. This can be shown by using (13)-(15). Note that a paraunitary matrix $\tau(z)$ becomes an orthogonal matrix, i.e., $\tau^{t} \tau=I$ if $\tau$ is not a function of $z$. The simplest orthogonal matrix that we will have occasion to consider is the "planar rotation" matrix $\boldsymbol{R}$ :

$$
\boldsymbol{R}=\left[\begin{array}{rr}
\cos \theta & \sin \theta \\
-\sin \theta & \cos \theta
\end{array}\right]
$$

Thus in the following equation:

$$
\left(\begin{array}{l}
V_{1} \\
V_{2}
\end{array}\right)=\boldsymbol{R}\left(\begin{array}{l}
U_{1} \\
U_{2}
\end{array}\right)
$$

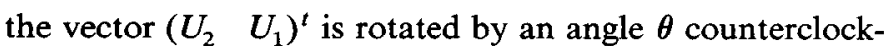
wise, to obtain the vector $\left(\begin{array}{ll}V_{2} & V_{1}\end{array}\right)^{t}$. This operation will be denoted as in Fig. 6.

\section{The Link Between The LBR Approach, WaVe Filters, AND ORTHOgONAL FILTERS}

In this section we begin with a basic LBR two-pair [9] and obtain an "orthogonal" implementation for this. The derivation simultaneously reveals the relation to wave "adaptors," and it becomes clear that the three families of filters are tied together by the "planar rotation circuit" of (17).

The most basic LBR two-pair (Type 1A) used in the synthesis of filters in [9] has the following transfer matrix:

$$
\tau(z)=\frac{\left[\begin{array}{cc}
1-\sigma & \sqrt{\sigma}\left(1+z^{-1}\right) \\
\sqrt{\sigma}\left(1+z^{-1}\right) & -(1-\sigma) z^{-1}
\end{array}\right]}{1+\sigma z^{-1}}
$$

\footnotetext{
${ }^{3}$ Superscript dagger $\left({ }^{\dagger}\right)$ stands for transposition followed by complex conjugation.
}

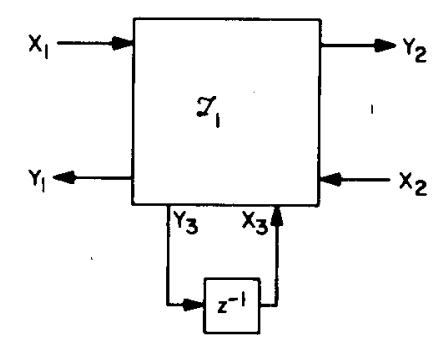

Fig. 7. Type 1A two-pair implementation.

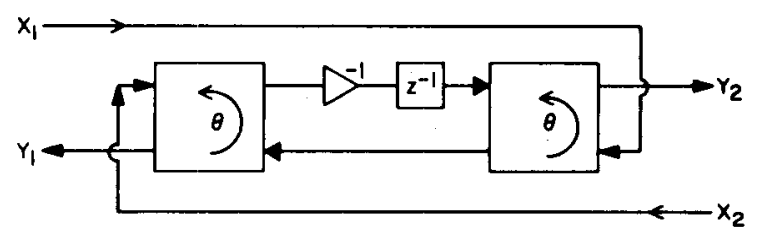

Fig. 8. Orthogonal implementation of Type 1A two-pair.

where $0<\sigma<1$. This two-pair is also obtainable by transforming continuous-time lossless elements into the digital scattering domain. This can be seen from the works of Constantinides and others. See, for example, [24],[25]. The contributions of Swamy and Thyagarajan [4] also clearly demonstrate this.

This two-pair can be implemented in the form shown in Fig. 7, where the $3 \times 3$ delay-free transfer matrix $\tau_{1}$ is

$$
\tau_{1}=\left[\begin{array}{ccc}
1-\sigma & \sigma & \sigma \\
1 & 0 & -1 \\
-(1-\sigma) & 1-\sigma & -\sigma
\end{array}\right]
$$

as shown in [9]. The matrix $\tau_{1}$ is closely related to the series wave adaptor [16]. Moreover, $\tau_{1}$ can be replaced with a normalized matrix $\boldsymbol{R}_{1}$ without affecting $\tau(z)$, where $\boldsymbol{R}_{1}$ is given by

$$
\boldsymbol{R}_{1}=\left[\begin{array}{ccc}
\sin ^{2} \theta & \cos \theta & \sin \theta \cos \theta \\
\cos \theta & 0 & -\sin \theta \\
-\sin \theta \cos \theta & \sin \theta & -\cos ^{2} \theta
\end{array}\right]
$$

where $\cos \theta=\sqrt{\sigma}, \sin \theta=\sqrt{1-\sigma} . R_{1}$ is an orthogonal matrix and can be decomposed into a product of two planar norm preserving rotations as follows:

$$
\boldsymbol{R}_{1}=\left[\begin{array}{ccc}
\cos \theta & -\sin \theta & 0 \\
0 & 0 & 1 \\
\sin \theta & \cos \theta & 0
\end{array}\right]\left[\begin{array}{ccc}
0 & 1 & 0 \\
-\sin \theta & 0 & -\cos \theta \\
\cos \theta & 0 & -\sin \theta
\end{array}\right]
$$

This leads to an orthogonal implementation of the LBR two-pair, as shown in Fig. 8. Essentially then, this is an implementation of a normalized wave adaptor in terms of planar rotations. Note the similarity of Fig. 8 to the circuits given in [17].

All the first-order two-pairs in [9] are obtainable from the Type 1A two-pair by transformations, and, therefore, have implementations similar to Fig. 8. The second order two-pairs of Type 2. [9] are obtained from Type 1 first-order two-pairs by replacing the delay with

$$
G(z)= \pm z^{-1} \frac{\left(\beta+z^{-1}\right)}{1+\beta z^{-1}}, \quad|\beta|<1
$$




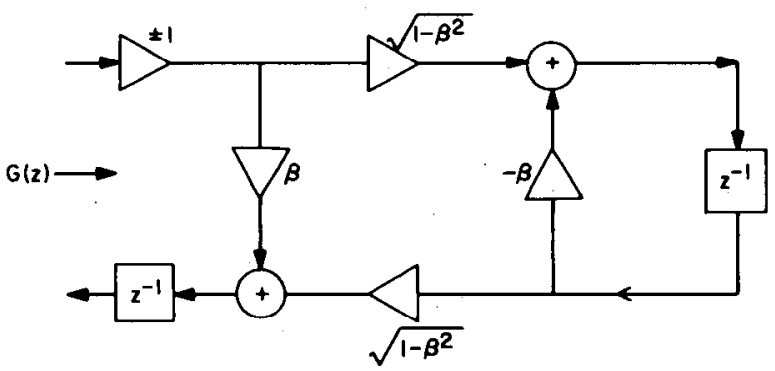

Fig. 9. Implementation of (22).

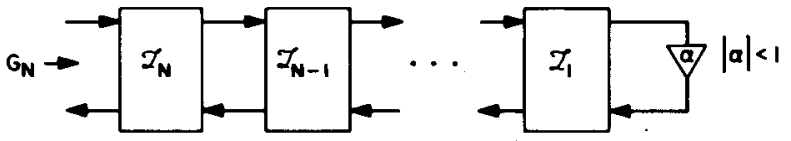

Fig. 10. Cascaded two-pair synthesis of a BR function.

which is itself an LBR function and can be implemented with planar rotators (Fig. 9). Type 2 two-pairs are characterized by two parameters, $\sigma$ and $\beta$, and are LBR if and only if $0<\sigma<1$ and $-1 \leqslant \beta \leqslant 1$. (Equation (22) represents a special case of spectral transformations in digital filters, advanced by Constantinides. See [23] and references therein.)

There is one more type of second-order LBR two-pair [9] that needs to be considered in order to complete the list of required two-pairs. We call this the Type 3 LBR two-pair. This has an analogy to the "Brune" section of the continuous-time domain. In the next section, we deal with this two-pair and show that it can be implemented with only two delays, and four planar rotations.

\section{IMPLEMENTATION OF THE TYPE 3 LBR Two-Pair and Relation to WaVE aND ORTHOGONAL FILTERS}

Let us briefly review the use of various LBR two-pairs, in the synthesis of a BR function $G_{N}(z)$ [9]. The synthesis consists of successive LBR two-pair extractions (Fig. 4), such that at each extraction step, the remainder BR function $G_{m-1}$ has an order less than that of the BR function $G_{m}$. The extraction scheme is based on the fact that if a BR function $G_{m}(z)$ attains the magnitude of unity at a frequency $\omega_{0}$ i.e., $\left|G_{m}\left(e^{j \omega_{0}}\right)\right|=1$, then there exists an LBR two-pair with transfer matrix $\tau_{m}(z)$, such that $G_{m-1}(z)$ is a lower order BR function. Moreover, if $\omega_{0}=0$ or $\pi$, then the LBR two-pair is of first order and the order of $G_{m-1}$ is one less. If $\omega_{0}$ is in the range $0<\omega_{0}<\pi$, then the LBR two-pair is of second order and the order of $G_{m-1}$ is two less. Consequently, by repeated extraction, one can synthesize a BR function as a cascaded two-pair (Fig. 10) with each two-pair being of order one or two.

If $\omega_{0}$ in the above discussion is in the range $0<\omega_{0}<\pi$, and if $G_{m}\left(e^{j \omega_{0}}\right)$ is real (i.e., equal to 1 or -1 ), then a Type 2 LBR two-pair is extracted. As indicated in Section III, such a two-pair can be implemented with three planar rotations. Next, if $G_{m}\left(e^{j \omega_{0}}\right)$ is complex, i.e., $G_{m}\left(e^{j \omega_{0}}\right)=e^{j \alpha}$ then a cascade of three two-pairs is used [9]: a first-order

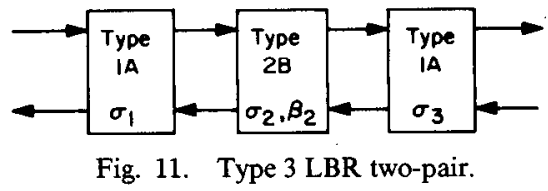

two-pair is followed by a second-order LBR two-pair, followed by another first-order two-pair. One of the firstorder two-pairs is LBR, while the other is not (i.e., it does not satisfy $0<\sigma<1$ ). However, the entire combination of the three two-pairs is indeed second-order LBR, and moreover can be implemented with two delays. One purpose of this section is to justify this. We also show an implementation that involves an orthogonal matrix, which in turn can be decomposed into four planar rotations. The resulting structure is identical to a building block in [17]. In addition the orthogonal matrix can be looked upon as a normalized version of the "Brune-adaptor" [18].

The second-order sections under consideration are of the form shown in Fig. 11, where a Type 1A two-pair has transfer matrix as in (18) and a Type 2B two-pair has transfer matrix:

$$
\tau(z)=\frac{\left[\begin{array}{cc}
(\sigma-1)\left(1+\beta z^{-1}\right) & \sqrt{\sigma}\left(1+2 \beta z^{-1}+z^{-2}\right) \\
\sqrt{\sigma}\left(1+2 \beta z^{-1}+z^{-2}\right) & -(\sigma-1) z^{-1}\left(\beta+z^{-1}\right)
\end{array}\right]}{1+\beta(1+\sigma) z^{-1}+\sigma z^{-2}}
$$

Let the $\sigma$ 's and $\beta$ 's of the three subsections be as indicated in Fig. 11. Then they are related [9] by

$$
\begin{aligned}
\left(1-\sigma_{1}\right)\left(1-\beta_{2}\right) \sigma_{3}+ & \sigma_{2}\left(1-\sigma_{3}\right)\left(1-\beta_{2}\right) \\
& +2\left(1-\sigma_{1}\right)\left(1-\sigma_{2}\right)\left(1-\sigma_{3}\right)=0 .
\end{aligned}
$$

This constraint essentially cancels an unintended "transmission zero" at $z=-1$. However, an actual digital implementation of this form invariably involves quantized versions of $\sigma$ 's and $\beta$ 's, and the constraint of (24) is not exactly satisfied. This results in spurious zeros or peaks in the synthesized transfer function. Such a difficulty is easily avoided by noting that the cascade of Fig. 11 is equivalent to the direct implementation of a second-order LBR twopair with chain matrix:

$$
\Pi(z)=\frac{\left[\begin{array}{cc}
1+r z^{-1}+s z^{-2} & t z^{-1}+u z^{-2} \\
u+t z^{-1} & s+r z^{-1}+z^{-2}
\end{array}\right]}{p-2 q z^{-1}+p z^{-2}}
$$

where $p$ and $q$ are such that $\operatorname{det} \Pi(z)=1$ (reciprocity condition). It is easily verified that $p$ and $q$ are given by

$$
4 p^{2}=\frac{(r+r s-t u)^{2}}{1+r^{2}+s^{2}-2 s-t^{2}-u^{2}}
$$

and

$$
q=-\left(\frac{1}{4} \cdot \frac{r+r s-t u}{s}\right) p
$$


Note that the corresponding transfer matrix is

$$
\tau(z)=\frac{\left[\begin{array}{cc}
u+t z^{-1} & \sqrt{s}\left[1+(r+r s-t u) / 2 s z^{-1}+z^{-2}\right] \\
\sqrt{s}\left[1+(r+r s-t u) / 2 s z^{-1}+z^{-2}\right] & -\left(t z^{-1}+u z^{-2}\right)
\end{array}\right]}{1+r z^{-1}+s z^{-2}} .
$$

In the rest of this paper we assume that the poles in (28) are complex conjugate, hence $s>0$. In Appendix A.2 we show that the parameters $r, s, t$, and $u$ can be computed as follows, for a given set of $\left(\sigma_{1}, \sigma_{2}, \sigma_{3}, \beta_{2}\right)$ :

Define the intermediate parameters:

$$
\begin{aligned}
a= & \sigma_{1} \sigma_{2} \sigma_{3} \\
b= & \beta_{2} \sigma_{1} \sigma_{3}\left(1+\sigma_{2}\right)+\left(\sigma_{1}+\sigma_{3}\right) \sigma_{2} \\
& +\beta_{2} \sigma_{3}\left(1-\sigma_{1}\right)\left(\sigma_{2}-1\right) \\
& +\sigma_{1}\left(1-\sigma_{3}\right)\left(\sigma_{2}-1\right)+\left(1-\sigma_{3}\right)\left(1-\sigma_{1}\right) \\
c= & \sigma_{1} \sigma_{3}+\beta_{2}\left(1+\sigma_{2}\right)\left(\sigma_{1}+\sigma_{3}\right)+\sigma_{2} \\
& +\left(1-\sigma_{1}\right)\left(\sigma_{2}-1\right)\left(\beta_{2}+\sigma_{3}\right) \\
& +\left(1-\sigma_{3}\right)\left(\sigma_{2}-1\right) \sigma_{1} \beta_{2}+\left(1-\sigma_{3}\right)\left(\sigma_{2}-1\right) \\
& +\left(1-\sigma_{3}\right)\left(1-\sigma_{1}\right) \beta_{2}\left(1+\sigma_{2}\right) \\
d= & \sigma_{1}+\sigma_{3}+\beta_{2}\left(1+\sigma_{2}\right)+\left(1-\sigma_{1}\right)\left(\sigma_{2}-1\right) \\
& +\beta_{2}\left(1-\sigma_{3}\right)\left(\sigma_{2}-1\right)+\left(1-\sigma_{1}\right)\left(1-\sigma_{3}\right) \sigma_{2} .
\end{aligned}
$$

Then compute

$$
r=\frac{b-2 a}{c+3 a-2 b} \quad s=\frac{a}{c+3 a-2 b} .
$$

It remains to compute $t$ and $u$. Recall that the extraction process is being done on a BR function $G_{m}(z)$ where $G_{m}\left(e^{j \omega_{0}}\right)=e^{j \alpha}$. Compute $t$ and $u$ from

$$
t \sin \omega_{0}+\sin \theta_{0}+r \sin \left(\theta_{0}-\omega_{0}\right)+s \sin \left(\theta_{0}-2 \omega_{0}\right)=0
$$

$u=-t \cos \omega_{0}+\cos \theta_{0}+r \cos \left(\theta_{0}-\omega_{0}\right)+s \cos \left(\theta_{0}-2 \omega_{0}\right)$.

It now remains to find a cannonic realization of the transfer matrix of (28). We show in Appendix A.3 that there exists a state-space realization:

$$
\begin{aligned}
\boldsymbol{x}(n+1) & =\boldsymbol{A} \boldsymbol{x}(n)+B \boldsymbol{u}(n) \\
y(n) & =C \boldsymbol{x}(n)+\mathrm{Du}(n)
\end{aligned}
$$

where

$$
\begin{aligned}
& A=\left[\begin{array}{cc}
\lambda_{1} & 0 \\
0 & \lambda_{2}
\end{array}\right], \quad B=\left[\begin{array}{cc}
\alpha_{1} & \alpha_{3} \\
\alpha_{4} / \alpha_{8} & 1
\end{array}\right] \\
& C=\left[\begin{array}{cc}
1 & \alpha_{7} \\
\alpha_{3} / \alpha_{1} & \alpha_{8}
\end{array}\right], \quad D=\left[\begin{array}{cc}
u & \sqrt{s} \\
\sqrt{s} & 0
\end{array}\right]
\end{aligned}
$$

with $\alpha_{k}$ 's defined according to

$$
\begin{aligned}
& \alpha_{1}=\frac{\lambda_{1}(t-r u)-s u}{\lambda_{1}-\lambda_{2}}, \quad \alpha_{2}=\frac{s u-\lambda_{2}(t-r u)}{\lambda_{1}-\lambda_{2}} \\
& \alpha_{3}=\frac{\lambda_{1}(r-r s-t u)-2 s(s-1)}{2 \sqrt{s}\left(\lambda_{1}-\lambda_{2}\right)}=\alpha_{5} \\
& \alpha_{4}=\frac{2 s(s-1)-\lambda_{2}(r-r s-t u)}{2 \sqrt{s}\left(\lambda_{1}-\lambda_{2}\right)}=\alpha_{7} \\
& \alpha_{6}=-\frac{u+\lambda_{1} t}{\lambda_{1}-\lambda_{2}}, \quad \alpha_{8}=\frac{u+\lambda_{2} t}{\lambda_{1}-\lambda_{2}}
\end{aligned}
$$

provided the $\alpha_{k}$ 's satisfy the following conditions:

$$
\alpha_{1} \alpha_{6}=\alpha_{3}^{2} \quad \alpha_{2} \alpha_{8}=\alpha_{4}^{2} .
$$

Assuming that $\tau(z)$ is LBR, it indeed turns out that the conditions of (36) are satisfied. This then shows that, for the LBR two-pair under consideration, the minimum degree (i.e., order of $\boldsymbol{A}$-matrix for a minimal realization) is 2 .

Obtaining an Orthogonal Realization of the LBR Two-Pair of (28)

The minimal realization of (34) in general has complex multipliers. We wish to obtain a more convenient realization by using a similarity transformation. Moreover, we would like to obtain a realization that involves planar rotations as the only computational units. This can be obtained by first deriving an "orthogonal" realization. We find it convenient to start by restating the LBR property of the transfer matrix $\tau(z)$ in terms of the state-space representation. To this end, let us begin by considering the circuit of Fig. 12. A multi-input multi-output system $\tau(z)$ is shown, with $A_{1}, B_{1}, C_{1}, D_{1}$ denoting a minimal statespace realization. We assume $A_{1}, B_{1}, C_{1}, D_{1}$ to be real. Let us now construct the following matrix:

$$
S_{1}=\left[\begin{array}{ll}
D_{1} & C_{1} \\
B_{1} & A_{1}
\end{array}\right] .
$$

Let us assume that $S_{1}$ is orthogonal, i.e., $S_{1}^{t} S_{1}=I$. This implies

$y^{t}(n) y(n)=u^{t}(n) u(n)+x^{t}(n) x(n)-x^{t}(n+1) x(n+1)$.

Assume that the input is zero for $n>\boldsymbol{N}$ where $\boldsymbol{N}>0$ is arbitrary. Summing from 0 to $N$ we get

$$
\begin{array}{r}
\sum_{n=0}^{N} y^{t}(n) y(n)=\sum_{n=0}^{N} u^{t}(n) u(n)+x^{t}(0) x(0) \\
-x^{t}(N+1) x(N+1) .
\end{array}
$$




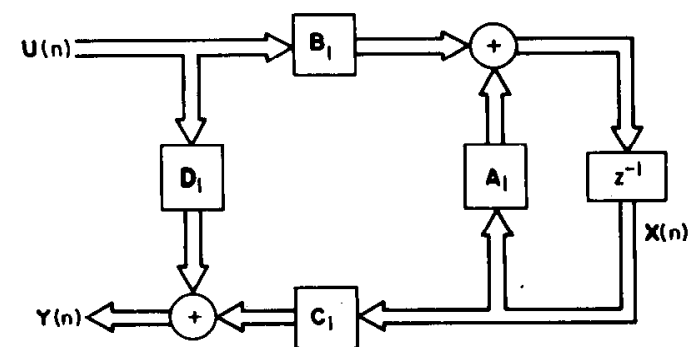

Fig. 12. State-space structure for $\tau(z)$.

From the orthogonality of $S_{1}$ we have

$$
A_{1}^{t} A_{1}-I=-C_{1}^{t} C_{1}
$$

By minimality assumption, $\left(C_{1}, A_{1}\right)$ is completely observable. Hence (40) provides us with a Lyapunov Equation, which clearly implies that $\tau(z)$ is stable. By using (40) in (39) it is easy to verify that

$$
\sum_{n=0}^{\infty} \boldsymbol{y}^{t}(n) y(n)=\sum_{n=0}^{N} \boldsymbol{u}^{t}(n) \boldsymbol{u}(n)+\boldsymbol{x}^{t}(0) \boldsymbol{x}(0)
$$

for the new realization. Thus for all input sequences that are zero for $n>N$, and for all possible $N>0$ we have

$$
\sum_{n=0}^{\infty} \boldsymbol{y}^{t}(n) \boldsymbol{y}(n)=\sum_{n=0}^{\infty} \boldsymbol{u}^{t}(n) \boldsymbol{u}(n)
$$

for zero initial conditions. This implies that the system $\tau(z)$ is LBR.

In summary, if the state-space realization is such that $S_{1}$ is orthogonal (i.e., LBR), then the transfer matrix $\tau(z)$ itself is LBR. Conversely, if $\tau(z)$ is LBR, then there always exists a minimal realization such that $S_{1}$ is orthogonal (for a proof see Appendix A.4). This leads to the discrete time version of the LBR lemma:

Discrete-Time LBR Lemma: Let $\tau(z)$ be a discrete-time transfer matrix and let $(A, B, C, D)$ be a minimal realization. Then $\tau(z)$ is LBR if and only if there exists a real symmetric positive definite matrix $\boldsymbol{P}$ such that

$$
\begin{aligned}
& D^{t} D+B^{t} P B=I \\
& C^{t} C+A^{t} P A=P \\
& C^{t} D+A^{t} P B=0 .
\end{aligned}
$$

An alternative proof of this lemma can be given by employing the bilinear transformation on the continuous-time version of the LBR lemma [12], which itself is the scattering domain version of the "Kalman-Yakubovich" lemma. The above proof is simpler and based entirely on $z$-domain concepts.

According to the above lemma, the transfer matrix of (28) can be realized in the form of Fig. 12, where matrix $S_{1}$

$$
S_{2}=\left[\begin{array}{cc}
k_{1} k_{2} & \hat{k}_{2} \hat{k}_{4} \\
-\hat{k}_{1} & 0 \\
-k_{1} \hat{k}_{2} k_{3} & k_{2} k_{3} \hat{k}_{4}+\alpha \hat{k}_{3} k_{4} \\
k_{1} \hat{k}_{2} \hat{k}_{3} & -k_{2} \hat{k}_{3} \hat{k}_{4}+\alpha k_{3} k_{4}
\end{array}\right.
$$

of (37) is now a $4 \times 4$ orthogonal matrix. Given such a state-space representation, if we now apply an orthogonal transformation:

$$
\begin{aligned}
& A_{2}=T^{-1} A_{1} T \\
& B_{2}=T^{-1} B_{1} \\
& C_{2}=C_{1} T \\
& D_{2}=D_{1}
\end{aligned}
$$

where $\boldsymbol{T}^{-1}=\boldsymbol{T}^{t}$, then the matrix

$$
S_{2}=\left[\begin{array}{ll}
D_{2} & C_{2} \\
B_{2} & A_{2}
\end{array}\right]
$$

continues to be orthogonal. It is computationally beneficial to apply transformations of this form in order to obtain a matrix $\boldsymbol{S}_{2}$ with larger number of null entries. In particular, if $\boldsymbol{T}$ is chosen as the following orthogonal matrix:

$$
\boldsymbol{T}=\left[\begin{array}{cc}
k & \sqrt{1-k^{2}} \\
\sqrt{1-k^{2}} & -k
\end{array}\right], \quad k=\frac{ \pm C_{21}}{\sqrt{C_{21}^{2}+C_{22}^{2}}}
$$

where $C_{i j}$ are the elements of $C_{1}$, this ensures that the $(2,2)$ element of $C_{2}$ is zero. Also recall the form of $D$ matrix (Eqn. (34)). Thus in summary, the LBR matrix of (28) can be realized in the form of Fig. 12, where the orthogonal matrix of (45) is of the form

$$
\boldsymbol{S}_{2}=\left[\begin{array}{cccc}
s_{11} & s_{12} & s_{13} & s_{14} \\
s_{21} & 0 & s_{23} & 0 \\
s_{31} & s_{32} & s_{33} & s_{34} \\
s_{41} & s_{42} & s_{43} & s_{44}
\end{array}\right]
$$

It only remains to factorize this orthogonal matrix into a product of planar rotations of the form in (17). This can be done in a systematic fashion, using standard techniques such as those described in [19]. In view of the two null entries in (47), it turns out to be possible to implement $\boldsymbol{S}_{2}$ with only four planar rotations. A convenient way to define the four factors is the following:

Let $k_{i}=\cos \theta_{i}$ and $\hat{k}_{i}=\sin \theta_{i}$. Let us construct the numbers $\left(k_{1}, \hat{k}_{1}, \cdots, k_{4}, \hat{k}_{4}\right)$ as follows:

$$
\begin{array}{ll}
k_{1}=s_{23}, \hat{k}_{1}=-s_{21} & k_{2}=\frac{s_{11}}{k_{1}}, \hat{k}_{2}=\sqrt{1-k_{2}^{2}} \\
k_{3}=\frac{-s_{33}}{\hat{k}_{1} \hat{k}_{2}}, \hat{k}_{3}=\frac{s_{43}}{\hat{k}_{1} \hat{k}_{2}} & k_{4}=\frac{s_{14}}{\hat{k}_{2}}, \hat{k}_{4}=\frac{s_{12}}{\hat{k}_{2}} .
\end{array}
$$

Then the orthogonal matrix of (47) can be written in the following form:

$$
\left.\begin{array}{cc}
\hat{k}_{1} k_{2} & \hat{k}_{2} k_{4} \\
k_{1} & 0 \\
-\hat{k}_{1} \hat{k}_{2} k_{3} & k_{2} k_{3} k_{4}-\alpha \hat{k}_{3} \hat{k}_{4} \\
\hat{k}_{1} \hat{k}_{2} \hat{k}_{3} & -k_{2} \hat{k}_{3} k_{4}-\alpha k_{3} \hat{k}_{4}
\end{array}\right]
$$

where $\alpha= \pm 1$ is the determinant of $S_{2}$. The matrix $S_{2}$ can 


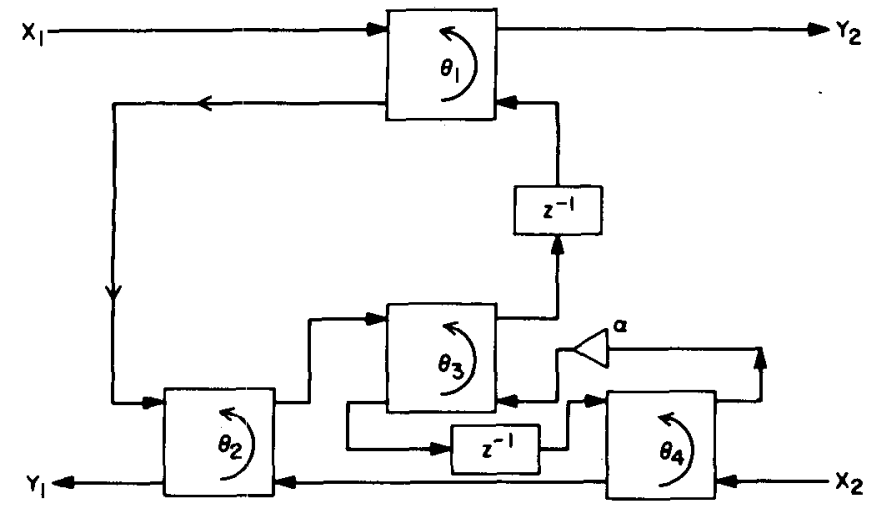

Fig. 13. Orthogonal implementation of the LBR two-pair of (28).

now be factored in terms of the four angles:

$$
\begin{aligned}
S_{2}= & {\left[\begin{array}{cccc}
1 & 0 & 0 & 0 \\
0 & 1 & 0 & 0 \\
0 & 0 & k_{3} & \alpha \hat{k}_{3} \\
0 & 0 & -\hat{k}_{3} & \alpha k_{3}
\end{array}\right]\left[\begin{array}{cccc}
k_{2} & 0 & \hat{k}_{2} & 0 \\
0 & 1 & 0 & 0 \\
-\hat{k}_{2} & 0 & k_{2} & 0 \\
0 & 0 & 0 & 1
\end{array}\right] } \\
& \cdot\left[\begin{array}{cccc}
1 & 0 & 0 & 0 \\
0 & 1 & 0 & 0 \\
0 & 0 & k_{4} & \hat{k}_{4} \\
0 & 0 & -\hat{k}_{4} & k_{4}
\end{array}\right]\left[\begin{array}{cccc}
k_{1} & 0 & \hat{k}_{1} & 0 \\
-\hat{k}_{1} & 0 & k_{1} & 0 \\
0 & 0 & 0 & 1 \\
0 & 1 & 0 & 0
\end{array}\right] .
\end{aligned}
$$

Thus an overall implementation of the LBR two-pair of (28) in terms of planar rotations is, as shown in Fig. 13. Once again, each rectangular box stands for a rotation matrix as described in (17). In summary, an orthogonal implementation of the LBR two-pair of (28) has been obtained. Notice that the structure of Fig. 13 is precisely the one advanced in [17].

We conclude this section by making the following observation. The results of the next section (Section $V$ ) are currently being generalized [26] for the synthesis of $m$-input $p$-output LBR matrices, in the form of an LBR matrix two-pair cascade. These results can also be used to rederive the results of this section (Section IV) in a simpler and elegant manner. In particular, the proof that the McMillan degree of the LBR two-pair of (28) is 2, and the proof that it can be implemented with four planar rotation operators, follow from this generalization [26] easily.

\section{Synthesis of $2 \times 1$ LBR Transfer Functions BY LBR MatriX Two-PaIr EXTraction. AN Approach to Pipelineable ORThogonal Filters}

Given a scalar BR transfer function $H(z)$ it can be realized in a "structurally bounded" manner, in a number of ways. For example, [20]:

Method 1. The transfer function $H(z)$ can be embedded into an LBR two-pair, which in turn is synthesized by factorizing the transfer matrix or chain matrix.

Method 2. $H(z)$ can be directly synthesized, without the embedding process, by LBR two-pair extraction.

Method 3. $H(z)$ can be embedded into a one-input two-output transfer matrix $G(z)([20$, p. 24]), which in turn can be synthesized by a "LBR matrix two-pair" extraction approach.

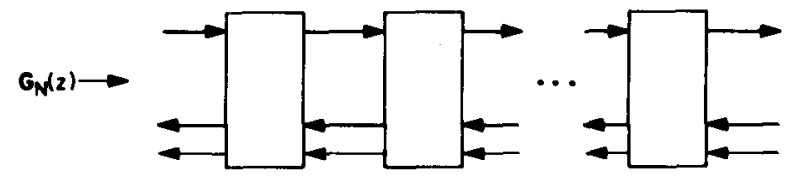

Fig. 14. Matrix-LBR two-pair cascade realization of $G_{N}(z)$.

The purpose of this section is to consider the details of Method 3. Thus given a BR function $H(z)=P(z) / Q(z)$, it can be embedded as one component of $G_{N}(z)=$ $[H(z) G(z)]^{t}$ where $G(z)=S(z) / Q(z)$, is related to $H(z)$ by

$$
\tilde{G}_{N}(z) G_{N}(z)=1
$$

i.e.,

$$
\tilde{H}(z) H(z)+\tilde{G}(z) G(z)=1
$$

If $\boldsymbol{G}_{N}(z)$, which is clearly an LBR vector, can be synthesized in such a way that it remains LBR (or BR) in spite of quantizations, then $H(z)$ remains $\mathrm{BR}$, and the resulting implementation has low passband sensitivity, according to Section II. (In this section, the subscript on $\boldsymbol{G}$ denotes order. Thus each component of $\boldsymbol{G}_{\boldsymbol{m}}(z)$ is a ratio of $m$ th degree polynomials in $z^{-1}$ ). This can be achieved by synthesizing $G_{N}(z)$ as a matrix-LBR cascade as in Fig. 14, provided the LBR two-pairs remain lossless (or passive) in spite of parameter quantization. The key step in the synthesis is, therefore, the LBR extraction of the type shown in Fig. 5. To be specific, given an LBR vector $G_{m}(z)=N_{m}(z) / D_{m}(z)$, we wish to extract an LBR matrix two-pair $\tau_{m}(z)$ such that the remainder $G_{m-1}(z)=$ $N_{m-1}(z) / D_{m-1}(z)$ is lower order LBR. Note that this synthesis problem is analogous to the scalar all-pass synthesis problem, leading to the Gray and Markel lattice $[6],[15]$.

We wish to proceed as follows: Given $\boldsymbol{G}_{m}(z)$, extract a constant LBR matrix two-pair $\hat{\tau}_{m}$ (i.e., a $3 \times 3$ orthogonal matrix) such that the remainder is of the form $z^{-1} G_{m-1}$, with $\boldsymbol{G}_{m-1}$ being of order $m-1$. Let $A_{m}, \boldsymbol{B}_{m}, C_{m}, D_{m}$ denote the (constant) chain parameters of $\hat{\tau}_{m}$. Then

$$
\begin{aligned}
D_{m}(z) & =A D_{m-1}(z)+z^{-1} B N_{m-1}(z) \\
N_{m} & =C D_{m-1}(z)+z^{-1} D N_{m-1}(z) .
\end{aligned}
$$

From the "extraction formula" (13), it is clear that a constant scale factor in the chain parameters does not affect the remainder. Let us, therefore, set the scalar $A=1$ without loss of generality. Also, assume $D_{m}(\infty)=D_{m-1}(\infty)$ $=1$, (i.e., the constant coefficient of the denominator polynomial is normalized to unity). From (52), we immediately get

$$
\boldsymbol{C}=\boldsymbol{G}_{m}(\infty)=\boldsymbol{k}_{m} \quad \text { (say) }
$$

Also, it is clear from ( 51) that $N_{m-1}(z)$ has order $\leqslant m-1$. It now remains to force an order reduction on $D_{m-1}(z)$, which is given by:

$$
D_{m-1}(z)=\left[D_{m}-B D^{-1} N_{m}(z)\right] /\left(1-B D^{-1} C\right) .
$$

By inspection of (54) it is obvious that this can be done by choosing $B$ and $D$ such that

$$
\left.z^{m} D_{m}(z)\right|_{z=0}=\left.B D^{-1} z^{m} N_{m}(z)\right|_{z=0} .
$$




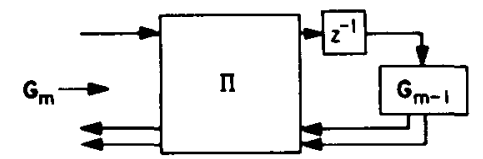

Fig. 15. Order-reduction of an $L B R$ vector.

In view of the LBR property of $G_{m}$, we have

$$
G_{m}^{t}(\infty) G_{m}(0)=1
$$

i.e.,

$$
\left.N_{m}^{t}(\infty) z^{m} N_{m}(z)\right|_{z=0}=\left.z^{m} D_{m}(z)\right|_{z=0} .
$$

Thus the choice $B=N_{m}^{t}(\infty) D$ achieves the desired order reduction. The matrix $D$ can be taken as $I$ without affecting the above reduction process. In summary, if the following chain matrix is extracted:

$$
\Pi=\left[\begin{array}{cc}
1 & \boldsymbol{k}_{m}^{t} \\
\boldsymbol{k}_{m} & \boldsymbol{I}
\end{array}\right]
$$

this results in a lower order remainder $\boldsymbol{G}_{m-1}(z)$. However, this chain matrix does not satisfy the paraunitary conditions of (15) and is, therefore, not LBR. This difficulty is easily overcome by scaling (58) such that the order reduction is not affected. One such scaling scheme results in the following chain matrix:

$$
\Pi_{m, 1}=\left[\begin{array}{cc}
1 / \sqrt{1-\boldsymbol{k}_{m}^{t} \boldsymbol{k}_{m}} & \boldsymbol{k}_{m}^{t}\left(\boldsymbol{I}-\boldsymbol{k}_{m} \boldsymbol{k}_{m}^{t}\right)^{-t / 2} \\
\boldsymbol{k}_{m} / \sqrt{1-\boldsymbol{k}_{m}^{t} \boldsymbol{k}_{m}} & \left(\boldsymbol{I}-\boldsymbol{k}_{m} \boldsymbol{k}_{m}^{t}\right)^{-t / 2}
\end{array}\right]
$$

where the matrix $\left(I-\boldsymbol{k}_{m} \boldsymbol{k}_{m}^{t}\right)$ has been factorized as

$$
\boldsymbol{I}-\boldsymbol{k}_{m} \boldsymbol{k}_{m}^{t}=\left(\boldsymbol{I}-\boldsymbol{k}_{m} \boldsymbol{k}_{m}^{t}\right)^{1 / 2}\left(\boldsymbol{I}-\boldsymbol{k}_{m} \boldsymbol{k}_{m}^{t}\right)^{t / 2}
$$

with the square root defined as

$$
\begin{aligned}
& \left(\boldsymbol{I}-\boldsymbol{k}_{m} \boldsymbol{k}_{m}^{t}\right)^{1 / 2} \\
& \quad=\left[\begin{array}{cc}
\sqrt{1-k_{1}^{2}} & 0 \\
-k_{1} k_{2} / \sqrt{1-k_{1}^{2}} & \sqrt{\left(1-k_{1}^{2}-k_{2}^{2}\right) /\left(1-k_{1}^{2}\right)}
\end{array}\right]
\end{aligned}
$$

where $\boldsymbol{k}_{m}=\left(\begin{array}{ll}k_{1} & k_{2}\end{array}\right)^{t}$. In view of the LBR property of $\boldsymbol{G}_{m}(z),(16)$ holds, and in particular $\boldsymbol{k}_{m}$ defined by (53) satisfies $\boldsymbol{k}_{m}^{t} \boldsymbol{k}_{m}<1$. Thus $\boldsymbol{I}-\boldsymbol{k}_{m} \boldsymbol{k}_{m}^{t}$ is positive definite, and all square roots in (61) are real. The situation is depicted in Fig. (15) where $G_{m-1}(z)$ is paraunitary. Moreover, it can be shown that $G_{m-1}(z)$ is stable (Appendix A.5) and hence LBR.

Now that the extraction step is complete, let us consider the transfer matrix corresponding to the chain matrix of (59):

$$
=\left[\begin{array}{cc}
\boldsymbol{k}_{m} & \left(\boldsymbol{I}-\boldsymbol{k}_{m} \boldsymbol{k}_{m}^{t}\right)^{1 / 2} \\
\sqrt{1-\boldsymbol{k}_{m}^{t} \boldsymbol{k}_{m}} & -\boldsymbol{k}_{m}^{t}\left[\left(\boldsymbol{I}-\boldsymbol{k}_{m} \boldsymbol{k}_{m}^{t}\right) /\left(1-\boldsymbol{k}_{m}^{t} \boldsymbol{k}_{m}\right)\right]^{-t / 2}
\end{array}\right] .
$$

This transfer matrix being orthogonal, can be factorized

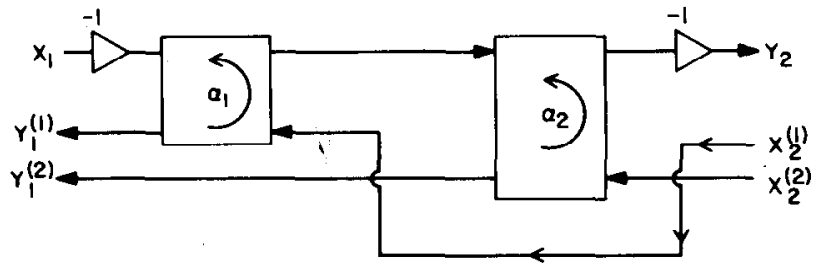

(a)

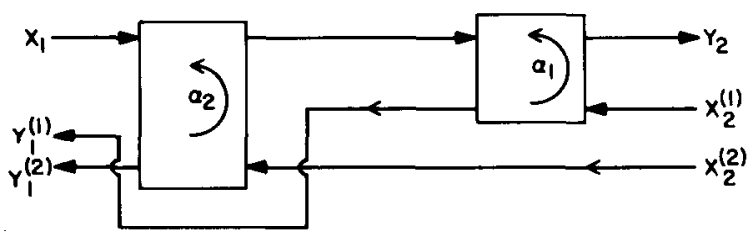

(b)

Fig. 16. (a) Implementation of $\tau_{m, 1}$. (b) Implementation of $\tau_{m, 2}$.

into planar rotations. Thus

$\begin{aligned} \tau_{m, 1} & =\left[\begin{array}{ccc}-\cos \alpha_{1} & \sin \alpha_{1} & 0 \\ \sin \alpha_{1} \cos \alpha_{2} & \cos \alpha_{1} \cos \alpha_{2} & \sin \alpha_{2} \\ \sin \alpha_{1} \sin \alpha_{2} & \cos \alpha_{1} \sin \alpha_{2} & -\cos \alpha_{2}\end{array}\right] \\ & =\left[\begin{array}{ccc}1 & 0 & 0 \\ 0 & \cos \alpha_{2} & \sin \alpha_{2} \\ 0 & \sin \alpha_{2} & -\cos \alpha_{2}\end{array}\right]\left[\begin{array}{ccc}-\cos \alpha_{1} & \sin \alpha_{1} & 0 \\ \sin \alpha_{1} & \cos \alpha_{1} & 0 \\ 0 & 0 & 1\end{array}\right](63)\end{aligned}$ where $k_{1}=-\cos \alpha_{1}$ and $k_{2}=\sin \alpha_{1} \cos \alpha_{2}$. It is interesting to find that the above orthogonal matrix, with $\alpha_{1}=\alpha_{2}=\theta$, reduces precisely to the matrix of (20) (after elementary row-column operations), which was shown to be related to the wave adaptor. Notice that the scaling of $\Pi(z)$ of (58) leading to an LBR matrix two-pair is not unique. Thus the following chain matrix is another scaled version, leading once again to a (different) reduced-order LBR remainder:

$$
\Pi_{m, 2}=\left[\begin{array}{cc}
1 / \sqrt{1-\boldsymbol{k}_{m}^{t} \boldsymbol{k}_{m}} & \boldsymbol{k}_{m}^{t} / \sqrt{1-\boldsymbol{k}_{m}^{t} \boldsymbol{k}_{m}} \\
\left(I-\boldsymbol{k}_{m} \boldsymbol{k}_{m}^{t}\right)^{-1 / 2} \boldsymbol{k}_{m} & \left(I-\boldsymbol{k}_{m} \boldsymbol{k}_{m}^{t}\right)^{-1 / 2}
\end{array}\right]
$$

The corresponding transfer matrix of the LBR two-pair is $\tau_{m, 2}$

$$
=\left[\begin{array}{cc}
{\left[\left(\boldsymbol{I}-\boldsymbol{k}_{m} \boldsymbol{k}_{m}^{t}\right) /\left(1-\boldsymbol{k}_{m}^{t} \boldsymbol{k}_{m}\right)\right]^{-1 / 2} \boldsymbol{k}_{m}} & \left(\boldsymbol{I}-\boldsymbol{k}_{m} \boldsymbol{k}_{m}^{t}\right)^{t / 2} \\
\left(1-\boldsymbol{k}_{m}^{t} \boldsymbol{k}_{m}\right)^{1 / 2} & -\boldsymbol{k}_{m}^{t}
\end{array}\right] .
$$

This orthogonal matrix has the following factorization in terms of planar rotations:

$$
\begin{aligned}
\tau_{m, 2}= & {\left[\begin{array}{ccc}
-\cos \alpha_{1} \sin \alpha_{2} & \sin \alpha_{1} & \cos \alpha_{1} \cos \alpha_{2} \\
\cos \alpha_{2} & 0 & \sin \alpha_{2} \\
\sin \alpha_{1} \sin \alpha_{2} & \cos \alpha_{1} & -\sin \alpha_{1} \cos \alpha_{2}
\end{array}\right] } \\
= & {\left[\begin{array}{ccc}
\sin \alpha_{1} & \cos \alpha_{1} & 0 \\
0 & 0 & 1 \\
\cos \alpha_{1} & -\sin \alpha_{1} & 0
\end{array}\right] } \\
& \cdot\left[\begin{array}{ccc}
0 & 1 & 0 \\
-\sin \alpha_{2} & 0 & \cos \alpha_{2} \\
\cos \alpha_{2} & 0 & \sin \alpha_{2}
\end{array}\right] .
\end{aligned}
$$

In Fig. 16, the implementations of $\tau_{m, 1}$ and $\tau_{m, 2}$ are shown. 


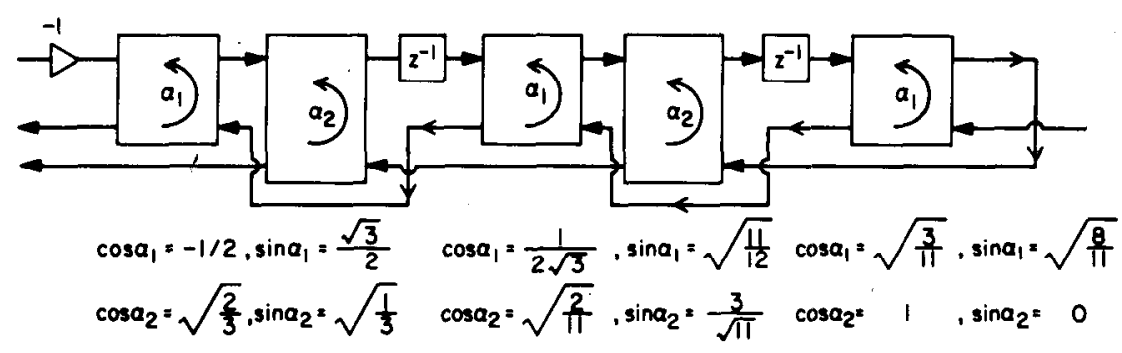

Fig. 17. Complete block-diagram for numerical example, based on the building block of Fig. 16(a).

The matrix $\tau_{m, 2}$ and its implementation in terms of planar rotations were first introduced in [7], where a " $b i$ channel" levinson algorithm is employed in order to derive the orthogonal filter implementation. The matrix $\tau_{m, 1}$ and study of the potentiality of the structure for VLSI implementations can be found in [8]. Note that in view of the delay unit separating successive LBR two-pairs (Fig. 15), these orthogonal filter structures are pipelineable [7],[8], and highly suited for VLSI. (In a broad sense, what we mean by pipelineability is that the computations that are required inside the filter can be arranged in such a way that every hardware unit representing a matrix two-pair can proceed with its computational task, without waiting for another computational unit to deliver its results, corresponding to the present sampling instant. For more precise and accurate statements, the reader is referred to [8], as exact details are beyond the scope of this paper.) Notice that the resulting filter structures consist entirely of the building blocks $\tau_{m, 1}$ (or $\tau_{m, 2}$ ), and lead to an extension of the cascaded lattice [6]. Accordingly, $\tau_{m, 1}$ and $\tau_{m, 2}$ are extensions of the Gray and Markel normalized lattice two-pair.

$A$ Numerical Example: Consider the synthesis of the following BR function:

$$
H(z)=\frac{\frac{1}{2}\left(1+\frac{1}{2} z^{-1}\right)}{1+\frac{3}{4} z^{-1}+\frac{1}{2} z^{-2}} .
$$

This can be embedded into the following LBR vector:

$$
G_{2}(z)=\frac{1}{1+\frac{3}{4} z^{-1}+\frac{1}{2} z^{-2}}\left[\begin{array}{c}
\frac{1}{2}\left(1+\frac{1}{2} z^{-1}\right) \\
\frac{1}{\sqrt{2}}\left(1+z^{-1}+z^{-2}\right)
\end{array}\right]
$$

The first LBR-extraction requires the $k$ parameter to be

$$
k^{(2)}=G_{2}(\infty)=\left(\begin{array}{ll}
\frac{1}{2} & \frac{1}{\sqrt{2}}
\end{array}\right)^{t}
$$

After extracting $\tau_{2,1}$ (as in (62)), the remainder all-pass vector is

$$
\boldsymbol{G}_{1}(z)=\frac{1}{\sqrt{3}}\left[\frac{\frac{1}{2}+z^{-1}}{1+\frac{1}{2} z^{-1}}\right] \cdot\left(\begin{array}{c}
-1 \\
\sqrt{2}
\end{array}\right)
$$

The second stage of LBR extraction requires

$$
k^{(1)}=G_{1}(\infty)=\frac{1}{2 \sqrt{3}}\left(\begin{array}{c}
-1 \\
\sqrt{2}
\end{array}\right)
$$

Once $\tau_{1,1}$ (equation (62)) is extracted, the final remainder is now

$$
\boldsymbol{G}_{0}(z)=\frac{1}{\sqrt{3}}\left(\begin{array}{c}
-1 \\
\sqrt{2}
\end{array}\right)
$$

which is a (constant) LBR vector. This vector can be implemented by rotations of the form (63) with the following parameter values:

$$
\begin{aligned}
& \cos \alpha_{1}=-G_{0,1}=\frac{1}{\sqrt{3}} \\
& \sin \alpha_{1}=\sqrt{1-G_{0,1}^{2}}=\sqrt{\frac{2}{3}} \\
& \cos \alpha_{2}=1 \\
& \sin \alpha_{2}=0 .
\end{aligned}
$$

Thus when the synthesis process terminates, we have a 0 -th order LBR vector as the final remainder, which itself can be represented as the "input-function" of an LBR matrix two-pair of the form (63) or (66), with suitable values of $\alpha_{1}$ and $\alpha_{2}$. Fig. 17 shows the overall implementation. Note also that the multipliers of values " -1 " in Fig. 16 can all be eliminated in the resulting cascade-structure of Fig. 17.

Inspection of (67) and Fig. 17 gives a first impression that there are too many multipliers in the structure. In general, an $N$ th-order transfer function (which can always be implemented with $2 N+1$ multipliers in direct form) requires $2 N+1$ planar rotations. As each rotation involves 4 multiplications, it appears that the structures involved require $8 N+4$ multiplications for an $N$ th-order transfer function. However, the planar rotations need not explicitly be implemented as a combination of $\mathbf{4}$ multipliers, as there are more efficient ways of doing it, such as the cordicalgorithms. Moreover, the structure of Fig. 17 has the advantages of low sensitivity, internal scaling, and freedom from limit cycles. The reader is referred to [7],[8], and [17] for details in this connection.

\section{Other Methods for Forcing Structural BOUNDEDNESS}

Given a BR function, there are several ways of finding structurally bounded implementations. So far, we have described three such methods (Section V). In this section, we wish to draw the reader's attention to other possible methods. Assume, for example, that a BR function $H(z)$ has been embedded into an LBR vector $G_{M}(z)$. Now 
consider a minimal state-space representation of $G_{M}(z)$ :

$$
\begin{gathered}
\boldsymbol{x}(n+1)=\boldsymbol{A} \boldsymbol{x}(n)+\boldsymbol{B} u(n) \\
\boldsymbol{y}(n)=\boldsymbol{C} \boldsymbol{x}(n)+\boldsymbol{D} u(n)
\end{gathered}
$$

where $\boldsymbol{G}_{M}(z)=\boldsymbol{Y}(z) / U(z)$. A minimal realization can always be found such that the matrix

$$
\boldsymbol{R}=\left[\begin{array}{ll}
\boldsymbol{D} & \boldsymbol{C} \\
\boldsymbol{B} & \boldsymbol{A}
\end{array}\right]
$$

is orthogonal i.e., LRR. This follows from the discrete-time version of the LBR Lemma (Section IV). The matrix $R$ can, therefore, be decomposed into a product of "planar rotations": $R=\boldsymbol{R}_{1} \boldsymbol{R}_{2} \boldsymbol{R}_{3} \cdots \boldsymbol{R}_{K}$, by means of systematic procedures (see, for example, [19]). Thus under a quantized environment $R$ can be retained to be (L)BR, hence $G_{M}(z)$ remains (L)BR, hence $H(z)$ remains $\mathrm{BR}$.

Yet another extremely simple means of achieving structural boundedness in certain transfer functions is the following: Consider again a BR function $H(z)$, as in (1). Assume that the numerator is a symmetric polynomial. Now define a transfer function $G(z)$ such that

$$
\left|G\left(e^{j \omega}\right)\right|^{2}+\left|H\left(e^{j \omega}\right)\right|^{2}=1 .
$$

If the numerator of $G(z)$ turns out to be an antisymmetric polynomial, then $H(z)$ can always be implemented in the following form:

$$
H(z)=\frac{1}{2}\left[A_{1}(z)+A_{2}(z)\right]
$$

where $A_{1}(z)$ and $A_{2}(z)$ are stable all-pass functions of orders $N_{1}$ and $N_{2}$, with $N=N_{1}+N_{2}$. Odd-order Butterworth, Chebyshev, and Elliptic transfer functions are among the many transfer functions that can be implemented as in (77). Now an all-pass function of order $N_{k}$ can be implemented cannonically, requiring only $N_{k}$ multipliers, by employing well-known techniques [6],[21]. Thus in spite of parameter quantization, the all-pass nature is preserved, hence the quantity $\left|H\left(e^{j \omega}\right)\right|$ can never exceed unity. This, therefore, leads to an elegant "structurally bounded" implementation requiring only $N$ multipliers. Note that, if an implementation involving only "planar-rotations" is required, it can be obtained by synthesizing each allpass function $A_{k}(z)$ as a cascade of the normalized latticestructure [6]. This also enables the suppression of limit cycles. The required number of planar rotations is only $N$ rather than $2 N+1$.

The justification for the above allpass decomposition can easily be given in the $z$-domain. However, it can also be understood by translating certain well-known properties from the theory of classical, doubly terminated filter synthesis (for example, see [22]).

All the filter structures considered so far are recursive, and implement IIR transfer functions. We conclude this section by mentioning that, for finite impulse response (FIR) digital filters, a method is outlined in [14] to achieve structural boundedness. The resulting structures have lowsensitivity property; to the best of our knowledge, this is the only class of structurally bounded implementations that cannot be related to the orthogonal implementations in terms of planar rotations in a natural way.

\section{Concluding Remarks}

A number of digital filter structures that are well-known for excellent properties under finite precision conditions have been rederived, based on the LBR approach. The results in this paper are not altogether surprising because passivity and losslessness are inherently present in all of these structures. The main contribution here is to obtain a derivation from a single viewpoint, so that all these fascinating results and structures can be understood in a unified manner, clearly placing in evidence the underlying relation between them.

\section{APPENDIX A.1}

Let $\boldsymbol{G}(z)$ be a BR vector. Based on elementary linear system concepts, we show $\boldsymbol{G}^{\dagger}\left(z_{0}\right) \boldsymbol{G}\left(z_{0}\right) \leqslant 1$ for all $z_{0}$ such that $\left|z_{0}\right|>1$. Note that, by definition of the BR property,

$$
\boldsymbol{G}^{\dagger}\left(e^{j \omega}\right) \boldsymbol{G}\left(e^{j \omega}\right) \leqslant 1, \quad \text { for all } \omega .
$$

Thus if $u(n)$ is the input sequence and $y(n)$ the output (vector) sequence, then this implies

$$
\boldsymbol{Y}^{\dagger}\left(e^{j \omega}\right) \boldsymbol{Y}\left(e^{j \omega}\right) \leqslant\left|U\left(e^{j \omega}\right)\right|^{2} .
$$

Integrating and applying Parseval's relation leads to

$$
\sum_{n=-\infty}^{\infty} \boldsymbol{y}^{t}(n) y(n) \leqslant \sum_{n=-\infty}^{\infty}|u(n)|^{2}
$$

for every input sequence with finite energy.

In particular let us consider the following finite-energy input:

$$
u(n)= \begin{cases}z_{0}^{n}, & -\infty \leqslant n \leqslant N \\ 0, & n>N\end{cases}
$$

where $N$ is any arbitrary integer, and $\left|z_{0}\right|>1$. Clearly

$$
\boldsymbol{y}(n)=\left\{\begin{array}{l}
\boldsymbol{G}\left(z_{0}\right) z_{0}^{n}, \quad n \leqslant N \\
\text { something else, } \quad n>N .
\end{array}\right.
$$

Thus

$$
\begin{aligned}
\sum_{n=-\infty}^{N} \boldsymbol{y}^{\dagger}(n) \boldsymbol{y}(n) & =G^{\dagger}\left(z_{0}\right) \boldsymbol{G}\left(z_{0}\right) \sum_{n=-\infty}^{N}\left|z_{0}\right|^{2 n} \\
& \leqslant \sum_{n=-\infty}^{\infty} \boldsymbol{y}^{\dagger}(n) \boldsymbol{y}(n) \leqslant \sum_{n=-\infty}^{\infty}|u(n)|^{2} \\
& =\sum_{n=-\infty}^{N}\left|z_{0}\right|^{2 n} .
\end{aligned}
$$

It immediately follows that $\boldsymbol{G}^{\dagger}\left(z_{0}\right) \boldsymbol{G}\left(z_{0}\right) \leqslant 1$. Moreover, this is a strict inequality unless $G(z)$ is constant.

\section{APPENDIX A.2 \\ Proof of the COMPUTATION RULES For THE PARAMETER $r, s, t, u$ IN SeCtION IV}

The cascaded two-pair of Fig. (11) has three sections. If the chain matrices are multiplied out and then the overall transfer matrix is computed, it has the following common denominator:

$$
D_{4}(z)=1+d z^{-1}+c z^{-2}+b z^{-3}+a z^{-4}
$$


where $a, b, c, d$ are as in (29). But there is a cancelation of the factor $\left(1+z^{-1}\right)^{2}$ between $D_{4}(z)$ and the $2 \times 2$ numerator matrix [9]: Dividing (A7) through by $\left(1+z^{-1}\right)^{2}$ we get the second-order denominator:

$$
D_{2}(z)=1+\frac{b-2 a}{c+3 a-2 b} z^{-1}+\frac{a}{c+3 a-2 b} z^{-2}
$$

which leads to the expressions in (30).

Next, note that the extraction of the two pair of (28) is done only under the condition that $G_{m}\left(e^{j \omega_{0}}\right)=e^{j \alpha}$. The overall two-pair is such that, at the frequency $\omega_{0}$, there is a transmission zero, i.e., zero of $T_{12}(z)\left[=T_{21}(z)\right]$. At this frequency, therefore, $G_{m}\left(e^{j \omega_{0}}\right)=T_{11}\left(e^{j \omega_{0}}\right)$. This leads to the two conditions (31) and (32).

\section{APPENDIX A.3}

Minimal Degree of The Two-Pair of (28)

For the two-pair under consideration, the $D$ matrix is clearly of the form in (34). If there exists (this existence assumption automatically gets verified in this appendix) a realization with a $2 \times 2 \boldsymbol{A}$ matrix, then there certainly exists a realization with the $A$ matrix as in (34). With $B=\left[b_{i j}\right]$ and $C=\left[c_{i j}\right]$, if we set

$$
\tau(z)=D+C(z I-A)^{-1} B
$$

then this leads to the following constraints on $B$ and $C$ :

$$
\begin{aligned}
r u+\alpha_{1}+\alpha_{2} & =t \\
s u-\lambda_{2} \alpha_{1}-\lambda_{1} \alpha_{2} & =0 \\
\alpha_{5}+\alpha_{7} & =\frac{r+r s-t u}{2 \sqrt{s}}-(\sqrt{s}) r \\
& =\frac{r-r s-t u}{2 \sqrt{s}} \\
\lambda_{1} \alpha_{7}+\lambda_{2} \alpha_{5} & =s \sqrt{s}-\sqrt{s} \\
\alpha_{3}+\alpha_{4} & =\frac{r+r s-t u}{2 \sqrt{s}}-r \sqrt{s} \\
& =\frac{r-r s-t u}{2 \sqrt{s}} \\
\lambda_{2} \alpha_{3}+\lambda_{1} \alpha_{4} & =s \sqrt{s}-\sqrt{s} \\
\alpha_{6}+\alpha_{8} & =-t \\
\lambda_{2} \alpha_{6}+\lambda_{1} \alpha_{8} & =u
\end{aligned}
$$

where the $\alpha_{k}$ 's are defined as

$$
\begin{array}{ll}
\alpha_{1}=c_{11} b_{11}, & \alpha_{2}=c_{12} b_{21} \\
\alpha_{3}=c_{21} b_{11}, & \alpha_{4}=c_{22} b_{21} \\
\alpha_{5}=c_{11} b_{12}, & \alpha_{6}=c_{21} b_{12} \\
\alpha_{7}=c_{12} b_{22}, & \alpha_{8}=c_{22} b_{22}
\end{array}
$$

from (A11), the constraints of (36) follow. The solutions (equation (35)) follow directly from (Ai10). It remains to show that the constraints of (36) hold for the LBR two-pair under consideration. (This is equivalent to showing that the minimal degree of the state-space realization is two, because the matrices $A, B, C, D$ have then been successfully constructed.) For this, we note that the paraunitary constraint on $\tau(z)$ of $(28)$ :

$$
\tau^{\prime}\left(z^{-1}\right) \tau(z)=I
$$

leads to the following constraint:

$$
4 s=\frac{(r+r s-t u)^{2}}{1+r^{2}+s^{2}-t^{2}-u^{2}-2 s} .
$$

In addition, each of $\lambda_{1}$ and $\lambda_{2}$ satisfies the following equation:

$$
1+r \lambda_{k}^{-1}+s \lambda_{k}^{-2}=0 .
$$

By making use of (A13) and (A14), it can be verified that (36) holds.

\section{APPENDIX A.4}

Assuming $\tau(z)$ is LBR, we show the existence of a minimal state-space realization $A_{0}, B_{0}, C_{0}, D_{0}$ such that the matrix

$$
\boldsymbol{S}_{0}=\left[\begin{array}{ll}
\boldsymbol{D}_{0} & \boldsymbol{C}_{0} \\
\boldsymbol{B}_{0} & \boldsymbol{A}_{0}
\end{array}\right]
$$

is orthogonal. Let $A, B, C, D$ be some minimal realization of $\tau(z)$. Then, because $\tau(z)$ is stable, we know there exists a real symmetric positive definite matrix $\boldsymbol{P}$ such that

$$
A^{t} P A-P=-C^{t} C \text {. }
$$

Letting $\boldsymbol{P}=\boldsymbol{T}^{-t} \boldsymbol{T}^{-1}$, and applying the similarity transformation $\boldsymbol{T}$ :

$$
\begin{aligned}
& A_{0}=T^{-1} A T \\
& B_{0}=T^{-1} B \\
& C_{0}=C T \\
& D_{0}=D
\end{aligned}
$$

we get a new minimal realization with

$$
A_{0}^{t} A_{0}+C_{0}^{t} C_{0}=I \text {. }
$$

Now, LBR property of $\tau(z)$ implies

$$
\sum_{0}^{\infty} y^{t} y(n)=\sum_{0}^{\infty} u^{t} u(n)
$$

for any finite energy input, assuming zero initial conditions. In particular, consider an input such that $u(n)=0$ for $n>N$, but arbitrary otherwise. Then

Hence,

$$
y(n)=C_{0} x(n), \quad n>N .
$$

$$
\begin{aligned}
& \sum_{n=N+1}^{\infty} y^{t} y(n)=\sum_{n=N+1}^{\infty} x^{t}(n) C_{0}^{t} C_{0} x(n) \\
& =\sum_{n=N+1}^{\infty} x^{t}(n)\left[I-A_{0}^{t} A_{0}\right] x(n) \\
& \sum_{n=N+1}^{\infty} \boldsymbol{y}^{t}(n) \boldsymbol{y}(n) \\
& =\sum_{n=N+1}^{\infty} \boldsymbol{x}^{t}(n) \boldsymbol{x}(n)-\sum_{n=N+1}^{\infty} \boldsymbol{x}^{t}(n+1) \boldsymbol{x}(n+1) \\
& =\boldsymbol{x}^{t}(N+1) \boldsymbol{x}(N+1) \text {. }
\end{aligned}
$$


Thus we get

$$
\sum_{n=0}^{N} \boldsymbol{y}^{t}(n) \boldsymbol{y}(n)=\sum_{n=0}^{N} \boldsymbol{u}^{t}(n) \boldsymbol{u}(n)-\boldsymbol{x}^{t}(N+1) \boldsymbol{x}(N+1)
$$

Repeating the above (A21) with $N$ replaced by $N-1$, and subtracting, we get

$$
\begin{aligned}
{\left[\begin{array}{ll}
y^{t}(N) & x^{t}(N+1)
\end{array}\right]\left[\begin{array}{c}
y(N) \\
\boldsymbol{x}
\end{array}\right.} \\
=\left[\begin{array}{ll}
\boldsymbol{u}^{t}(N) & \boldsymbol{x}^{t}(N)
\end{array}\right]\left[\begin{array}{l}
\boldsymbol{u}(N) \\
\boldsymbol{x}(N)
\end{array}\right]
\end{aligned}
$$

for all $N$. This implies that the matrix $S_{0}$ is orthogonal, which proves the desired result.

\section{APPENDIX A.5}

Proof of Stability of $\boldsymbol{G}_{\boldsymbol{m}-1}$ in Section V

Assuming that $G_{m}(z)$ is a BR vector, and assuming that the two-pair with chain matrix of $(58)$ is extracted, where $\boldsymbol{k}_{m}=\boldsymbol{G}_{\boldsymbol{m}}(\infty)$, let us first show that the corresponding remainder $\hat{G}_{m-1}(z)$ is stable. We know

$$
\hat{\boldsymbol{G}}_{m-1}(z)=\left[\boldsymbol{I}-\boldsymbol{G}_{m} \boldsymbol{k}_{m}^{t}\right]^{-1}\left[\boldsymbol{G}_{m}-\boldsymbol{k}_{m}\right] .
$$

At a pole $z_{0}$ of $\hat{G}_{m-1}(z)$, we have

$$
\operatorname{det}\left[\boldsymbol{I}-\boldsymbol{G}_{m} \boldsymbol{k}_{m}^{t}\right]=0
$$

which implies the existence of a non-null vector $v$ such that:

$$
\boldsymbol{v}^{\boldsymbol{t}} \boldsymbol{v}=\boldsymbol{v}^{t} \boldsymbol{k}_{m} \boldsymbol{G}_{m}^{\dagger}\left(z_{0}\right) \boldsymbol{G}_{m}\left(z_{0}\right) \boldsymbol{k}_{m}^{t} \boldsymbol{v}
$$

Assuming that $\left|z_{0}\right|>1$, let us bring about a conflict, which is equivalent to establishing that $\hat{\boldsymbol{G}}_{m-1}(z)$ is stable. Wc know, $\left|z_{0}\right|>1$ implies

Hence

$$
G_{m}^{\dagger}\left(z_{0}\right) G_{m}\left(z_{0}\right)<1 .
$$

$$
v^{t} v<v^{t} k_{m} k_{m}^{t} v
$$

But the matrix $\boldsymbol{I}-\boldsymbol{k}_{m} \boldsymbol{k}_{m}^{t}$ is real symmetric positive definite because $\boldsymbol{k}_{m}^{t} \boldsymbol{k}_{m}=\boldsymbol{G}_{m}^{\dagger}(\infty) \boldsymbol{G}_{m}(\infty)<1$ by LBR property of $G_{m}(z)$. Thus (A27) cannot be true.

This proves that $\hat{G}_{m-1}(z)$ cannot have a pole outside the unit circle. If there were a pole $z_{0}$ on the unit circle, this would imply that $\boldsymbol{I}-\boldsymbol{k}_{m} \boldsymbol{k}_{m}^{\boldsymbol{t}}$ is singular, which again, is not true in view of $\boldsymbol{G}_{m}^{\dagger}(\infty) \boldsymbol{G}_{m}(\infty)<1$. In summary, all poles of $\hat{\boldsymbol{G}}_{m-1}(z)$ are strictly inside the unit circle.

Now the actual remainder $z^{-1} G_{m-1}$ is related to $\hat{G}_{m-1}$ by a constant matrix, because the actual chain matrix of the LBR two-pair (59) or (64) is obtained from (58) by scaling. Thus $G_{m-1}$ has all poles strictly inside the unit circle.

\section{APPENDIX A.6}

\section{Matrix Two-PaIrs and EXTRACTION Formulas}

For a matrix two-pair, the vector signals $X_{1}, Y_{1}, X_{2}, Y_{2}$ are related by the chain parameter matrices $A, B, C, D$ as

$$
\begin{gathered}
X_{1}=A Y_{2}+B X_{2} \\
Y_{1}=C Y_{2}+D X_{2}
\end{gathered}
$$

or equivalently, by transfer parameter matrices $T_{i j}$ by

$$
\begin{aligned}
& Y_{1}=T_{11} X_{1}+T_{12} X_{2} \\
& Y_{2}=T_{21} X_{1}+T_{22} X_{2}
\end{aligned}
$$

Note that, for simplicity, functional dependence on $z$ is not explicitly shown. In order to derive the relations of (11), note that with $X_{2}=0$ we get

$$
X_{1}=A Y_{2}, \quad Y_{2}=T_{21} X_{1}
$$

hence

$$
T_{21}=A^{-1}
$$

provided $\boldsymbol{A}$ is square and has full normal rank. Moreover,

$$
Y_{1}=C Y_{2}=C A^{-1} X_{1}
$$

hence

$$
T_{11}=C A^{-1} \text {. }
$$

Similarly, by setting $\boldsymbol{X}_{1}=0$, we can derive the other two relations in (11). The relations in (12) follow from (11) directly.

Extraction Formulas: Now refer to Fig. 4 for two-pair extraction, and assume that all signals are vectors. We have

$$
\boldsymbol{X}_{2}=\boldsymbol{G}_{m-1} \boldsymbol{Y}_{2}
$$

Hence, from (A29)

$$
Y_{1}=C Y_{2}+D G_{m-1} Y_{2}=\left(C+D G_{m-1}\right) Y_{2} .
$$

From (A28) and (A36),

$$
X_{1}=\left(A+B G_{m-1}\right) Y_{2} .
$$

Equations (A37) and (A38) yield

$$
Y_{1}=\left(C+D G_{m-1}\right)\left(A+B G_{m-1}\right)^{-1} X_{1}
$$

assuming that $\boldsymbol{A}+\boldsymbol{B} \boldsymbol{G}_{\boldsymbol{m}-1}$ has full normal rank. Since $\boldsymbol{G}_{\boldsymbol{m}}$ is defined by $\boldsymbol{Y}_{1}=\boldsymbol{G}_{m} \boldsymbol{X}_{1}$, (14) follows from (A39). Next, (13) is obtained directly by inverting the relation in (14).

\section{Proof of Equivalence of (15) and the Paraunitary Property}

The transfer matrix $\tau(z)=\left[\boldsymbol{T}_{i j}(z)\right]$ being paraunitary satisfies $\tilde{\tau} \tau=I$, i.e.,

$$
\begin{gathered}
\tilde{T}_{11} T_{11}+\tilde{T}_{21} T_{21}=I \\
\tilde{T}_{12} T_{12}+\tilde{T}_{22} T_{22}=I \\
\tilde{T}_{11} T_{12}+\tilde{T}_{21} T_{22}=0
\end{gathered}
$$

(Recall that $\tilde{\boldsymbol{T}}_{11}(z)$ is abbreviation for $\boldsymbol{T}_{11}^{t}\left(z^{-1}\right)$ and so on.) By applying the relations of (11) in (A40), we immediately get

$$
\tilde{C} \boldsymbol{C}+I=\tilde{A} \boldsymbol{A} .
$$

Next, from (A41) we get, after simplification with the help of (A43),

$$
\tilde{\boldsymbol{D}} \boldsymbol{D}+\tilde{\boldsymbol{B} B}-\tilde{\boldsymbol{B}} \tilde{\boldsymbol{A}}^{-1} \tilde{\boldsymbol{C}} \boldsymbol{D}-\tilde{\boldsymbol{D}} \boldsymbol{C} \boldsymbol{A}^{-1} \boldsymbol{B}=\boldsymbol{I} .
$$

Similarly (A42) implies, with the help of (A43),

$$
\tilde{C} D=\tilde{A} B \text {. }
$$


By using (A45) in (A44), we finally arrive at

$$
\tilde{\boldsymbol{D} D}=\tilde{\boldsymbol{B}} \boldsymbol{B}+\boldsymbol{I} .
$$

Equations (A43), (A45), and (A46) establish that (15) holds for any paraunitary matrix two-pair.

Conversely, it can be shown that, if (15) holds, then the matrix two-pair is paraunitary (i.e., (A40), (A41), and (A42) then follow). The details of the converse are omitted in the interests of brevity.

\section{ACKNOWLEDGMENT}

The author wishes to acknowledge the pioneering contributions of Profs. A. Fettweis and P. Dewilde over the years, which motivated him towards the writing of this paper. Recent contributions by Henrot and Mullis [7] and by Rao and Kailath [8] were also similar motivations.

The author also wishes to thank Prof. S. K. Mitra of the University of California, Santa Barbara, for constant encouragement. Finally, the stimulating comments by the reviewers are gratefully acknowledged.

\section{REFERENCES}

[1] A. Fettweis, "Digital filter structures related to classical filter networks," Arch. Elek. Ubertragung., vol. 25, pp. 79-81, Feb. 1971.

[2] A. Fettweis, "Pseudopassivity, sensitivity, and stability of wave digital filter," IEEE Trans. Circuit Theory, vol. 19, pp. 668-673, Nov. 1972.

[3] A. Fettweis and K. Merrkotter, "Suppression of parasitic oscillations in wave filters," IEEE Trans. Circuits Syst., vol. 22, pp. 239-246, Mar. 1975.

[4] M. N. S. Swamy and K. Thyagarajan, "A new type of wave digital filters," J. Franklin Inst., vol. 300, no. 1, pp. 41-58, July 1975.

[5] P. Dewilde and E. Deprettere, "Orthogonal cascade realization of real multiport digital filters," Int. J. Circuit Theory Appl., vol. 8, 245-277, 1980.

[6] A. H. Gray, Jr. and J. D. Markel, "A normalized digital filter structure," IEEE Trans. Acoustics, Speech, Signal Processing, vol. ASSP-23, pp. 268-277, Junc 1975.

[7] D. Henrot and C. T. Mullis, "A modular and orthogonal digital filter structure for parallel processing," in IEEE Int. Conf. on Acoustics, Speech, and Signal Processing, pp. 623-626, Apr. 1983

[8] S. K. Rao and T. Kailath, "Orthogonal digital filters for VLSI implementation," IEEE Trans. Circuits Syst., vol. 31, pp. 933-945, Nov. 1984
[9] P. P. Vaidyanathan and S. K. Mitra, "Low passband sensitivity, digital filters: A generalized viewpoint and synthesis procedures," Proc. IEEE, pp. 404-423, April 1984.

[10] R. W. Newcomb, Linear Multiport Synthesis. New York: McGraw Hill, 1966.

[11] V. Belevitch, Classical Network Synthesis, San Francisco, CA: Holden-Day, 1968

[12] B. D. O. Anderson and S. Vongpanitlerd, Network Analysis and Synthesis. Englewood Cliffs, NJ: Prentice Hall, 1973.

[13] P. P. Vaidyanathan and S. K. Mitra, "Passivity properties of low sensitivity digital filter structures," IEEE Trans. Circuits Systems, vol. C.AS-32, pp. 217-224, Mar. 1985.

[14] "Very low-sensitivity FIR filter implementation using 'structural passivity' concept," IEEE Trans. Circuits Syst., vol. CAS-32, pp. 360-364, Apr. 1985.

[15] - S. K. Mitra, P. S. Kamat, and D. C. Huey, "Cascaded lattice realization of digital filters," Int. J. Circuit Theory Appl., vol. 5, pp. 3-11, 1977.

[16] R. E. Crochiere, "Digital ladder structures and coefficient sensitivity," IEEE Trans. Audio Electroacoust., vol. 20, pp. 240-246, Oct. 1972 .

[17] E. Deprettere, "Synthesis and fixed-point implementation of pipelined true orthogonal filters," in IEEE Int. Conf. on Acoustics, Speech, and Signal Processing, pp. 217-220, Boston, MA, 1983.

[18] G. O. Martens, "Wave digital adaptors for reciprocal second-order sections," IEEE Trans. Circuits Syst., vol. 25, pp. 1077-1082, Dec. 1978.

[19] F. D. Murnaghan, The Unitary and Rotation Groups. Washington, DC: Spartan Books, 1962.

[20] P. P. Vaidyanathan, "A general theory and synthesis procedure for low sensitivity digital filters structures," Ph.D. dissertation, Dep. of Elect. and Computer Eng., Univ. of California, Santa Barbara, June 1982.

[21] S. K. Mitra and K. Hirano, "Digital allpass networks," IEEE Trans. Circuits Syst., vol. 21, pp. 688-700, Sept. 1974.

[22] L. Gazsi, "Explicit formulae for multiplier values in lattice wave digital filters," in Second European Signal Processing Conf., Erlangen, W, Germany, pp. 99-102, 1983.

[23] A. G. Constantinides, "Spectral transformations for digital filters," Proc. Inst. Elec. Eng., vol. 117, pp. 1585-1590, Aug. 1970.

[24] A. G. Constantinides, "Alternative approach to design of wave digital filters," Electron. Lett., vol. 10, pp. 59-60, 1974.

[25] S. S. Lawson and A. G. Constantinides, "The design of digital filters using linear transformations on classical $L C$ two-ports," Proc. Network Theory Symp., pp. 369-378, 1975

[26] P. P. Vaidyanathan and S. K. Mitra, "A general family of multivariable digital lattice filters," in preparation

[27] D. A Vaughan-Pope and L. T. Bruton, "Transfer function synthesis using generalized doubly terminated two-pair networks," IEEE Trans. Circuits Syst., vol. 24, pp. 79-88, Feb. 1977.

[28] B. Friedlander, "Lattice filters for adaptive processing," Proc. IEEE, vol. 70, pp. 829-867, Aug. 1982.

[29] A: Fettweis, "On sensitivity and roundoff noise in wave digital filters," IEEE Trans. Acoustics, Speech, and Signal Proc., vol. 22, pp. 383-384, Oct. 1974.

\section{*}

P. P. Vaidyanathan (S'80-M'83), for a photograph and biography please see page 224 of the March 1985 issue of this Transactions. 